\title{
STORY BASED REPRESENTATION FOR BROADCASTED SPORTS VIDEO AND AUTOMATIC STORY SEGMENTATION
}

\author{
Naoko Nitta, Noboru Babaguchi, and Tadahiro Kitahashi
}

\author{
ISIR, Osaka University \\ 8-1 Mihogaoka Ibaraki, Osaka 567-0047, Japan \\ E-mail: \{naoko,babaguchi,kitahashi\}@am.sanken.osaka-u.ac.jp
}

\begin{abstract}
This paper presents a model to represent a broadcasted sports video in a semantical way and proposes a method to segment the sports video into the semantical units for the representation. Representation of a video should clarify its semantical content as accurately as possible. Our model structurizes the video and gives the suitable semantical descriptions to its particular time locations based on the structures of the sports video. We consider the speech transcript as the useful information stream for the generation of the semantical descriptions. The proposed method tries to segment the speech transcript into the units of the structurization in a probabilistic framework based on Bayesian networks. Moreover, the association with the image stream enables us to find the corresponding video segments. In this paper, we discuss some experimental results.
\end{abstract}

\section{INTRODUCTION}

Continuous increase in the amount of multimedia data has strongly required simple but meaningful representation for efficient multimedia applications. Aiming at the effective retrieval and the summarization system, this paper proposes how to represent a broadcasted sports video (hereafter simply called a sports video) in a clear and concise way, and presents a method to automatically segment the video into the units for the proposed representation.

Videos have some typical structures which depend on their genres. For instance, a news video can be considered as a sequence of units which start with an image frame presenting an anchor person followed by a variety of news. A drama video can be considered as an assembly of the semantically interrelated units with the in-between commercial message(CM) breaks. Such flows of the units construct every video and give it the semantical meaning or the story. Therefore, in order to represent video effectively, the structure of the video should be the key to grasp the story of the video. Considering such unit as the fundamental story unit, segmenting the video into these units and understanding their content must be done as a step toward semantical representation. In this paper, we discuss the structure of the sports video and propose a representation model which can be useful to understand its story.

Broadcasted video data consists of several multimodal information streams closely related to one another: image, audio, and text streams. The image stream is usually used temporally, since its low level feature helps us to find the content boundaries of the video. It is also used to get the semantical content, although the audio or the text stream can also be the effective source to get the information about the content. Therefore, the content of broadcasted video is generally analyzed by integrating several information streams. We call this method intermodal collaboration $[3,5]$.

There has been some prior work aiming at sports video content analysis with the intermodal collaboration $[1,2$, $3,7]$. Lazarescu et al. [1] tried to make annotation about the movement of the player by searching keywords from the text stream and analyzing the image stream. Chang et al. [2] tried to detect important events by integrating the audio and the image streams. Babaguchi et al. [3] also proposed event scene detection by integrating the text and the image streams. Zhong et al. [7] focused on the content structure of a sports video and tried to extract some patterned event boundaries from the image stream.

This paper focuses on the text stream which is the speech transcript of the announcers, and present a method of segmenting it into the units of the sports program, parts of which have useful semantical information to grasp the story, on the basis of the probabilistic Bayesian Network framework. Since a set of these units construct the unit of the sports game, finding the boundary of the sets leads us to segment the text stream into story units. Finally, we associate them with the image stream and realize the semantical video segmentation. In what follows, we deal with American Football games as a case study.

\section{REPRESENTATION MODEL FOR SPORTS VIDEO}

First of all, we consider how we describe a broadcasted sports video. Representation of the video should be concise but informative enough to cover the story. In this section, we discuss what kind of descriptions are needed to capture 


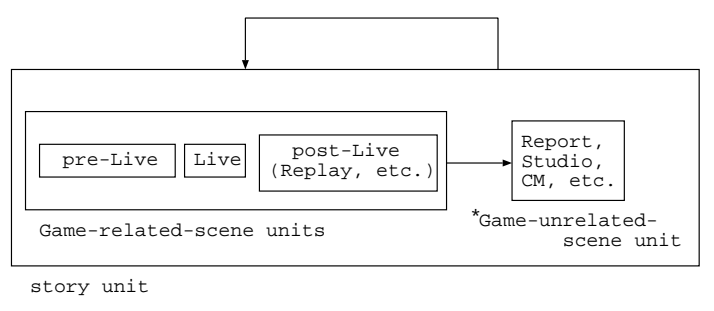

Figure 1: Structure of Sports TV Program. The “*” attached to the "Game-unrelated-segment" indicates the possibility of 0 occurrence of this segment.

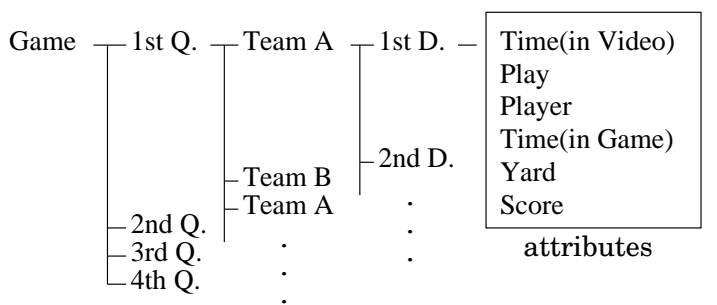

Figure 2: Structure of Sports Game

the story focusing on the structure of a sports TV program and a sports game.

- Structure of Sports TV Program

A TV program of a sports game can be regarded as a sequence of the scene units shown in Figure1. We define the "Live" scene as the time interval which begins with players starting to move and ends with an event stopping the flows of the game (e.g. the score event, the ball outbound, etc.), the "Pre-Live" and the "PostLive" scenes as the one before and after the "Live" scene which have some relationship to the in-between "Live" scene. These three scenes altogether construct a segment which matches a play. That is, a play can be defined as the interval since the players begin to move until an event occurs. Other scenes such as "Report" and "CM" are considered unrelated to the game. We regard the time interval between a "Live" scene and the next "Live" scene as a story unit which is the element of the whole story.

- Structure of Sports Game

A sports game in the case of American Football can be expressed as a tree, as shown in Figure2. It is actually going on by repeating the fundamental element of the tree, such as "1st Down" and "2nd Down" in Figure2. These fundamental elements can be viewed as basic logical units describing a sports game and correspond to the story unit of the sports program. The information needed to explain the sub-story of each story unit is about its upper node(1st-4th Quarter, offense team name, 1st-4th Down) and about the unit itself(the attributes such as play, player, time-in-game, score, etc.). Although Figure2 shows the structure of American football game, it is noted that such a tree-like structure is general to many kinds of sports.

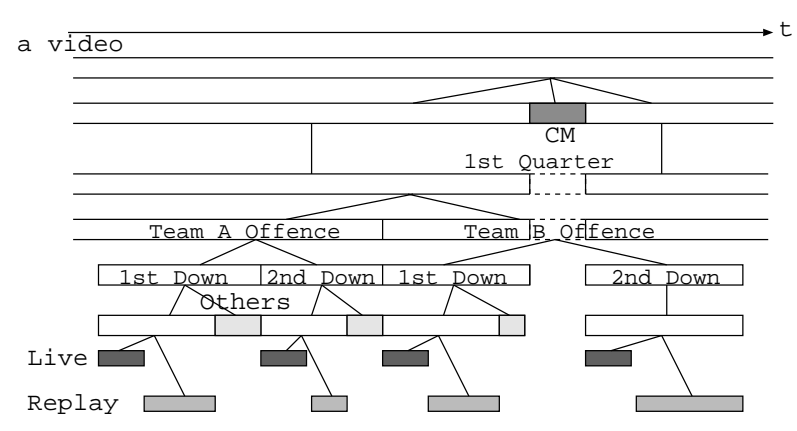

Figure 3: Overall Structure of Sports Video

Considering these two structures, we represent the sports video as shown in Figure3. In words, we can segment the whole sports video into the story units and structure it in a tree corresponding to the sports game. Further, the segmented story unit can be segmented into the scene units of the sports TV program. Finally, generating the semantical descriptions about the sub-story of each unit should adequately represent the story of the video.

\section{SCENE AND STORY SEGMENTATION}

Videos have several sources of information such as image, text, and audio streams. The image stream is usually considered to be useful for segmentation of the video. Some researchers $[2,5,7,8]$ tried to detect the boundary of the story unit by using the image features of the sports video, such as the stationary images seen at the beginning of a play. However, since the announcers talk about the situation of the game in a sports game program, the semantical content of the video can be extracted much more easily and effectively from the speech. Some researchers $[2,3,4,5,6]$ proposed to get the semantical information from the speech transcript obtained from speech recognition or encoded ClosedCaption. Most of them used some domain-specific key words and tried to segment and index the sports/news video semantically. However, most of the work left the semantical structure of the video out of consideration and we prefer less key words to determine beforehand. In this paper, considering the semantical structure of the sports video, we try to segment the text stream, which is the speech transcript, into the scene units of the TV program, then form the story unit by assembling these segmented scene units, evading the use of many domain-specific key words.

We use the text stream called Closed-Caption(CC) as the transcript of the speech and the sound. It also contains several markers, such as " $>>$ " to indicate the change of the speaker, "NAME:" to indicate the speaker, etc. It is embedded in the video signals as the character code and is easy to be reproduced in the text form. We add the "Timed-Stamp", which represents the image frame in which the first character of each line appears, to the original CC text.

\subsection{Segmentation of Text Stream}

The CC text and the image streams usually have the time-lag between them. Moreover, the announcers do not necessarily 


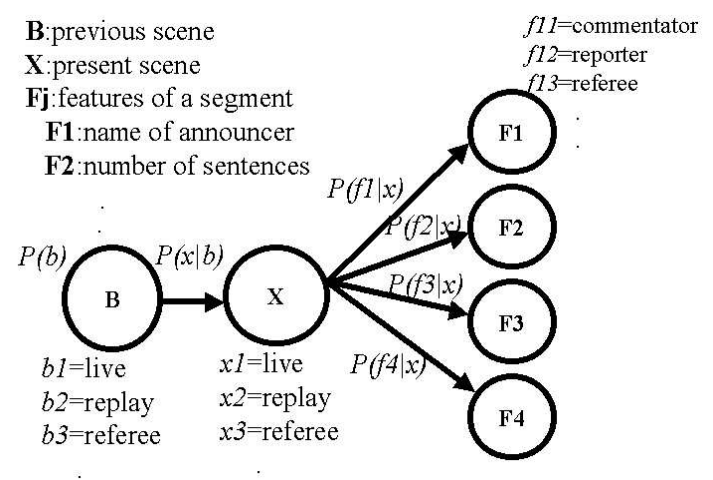

Figure 4: Bayesian Network

talk about the present scene. Therefore, the CC text should be segmented separately from the video stream so that the segments correspond to the video scenes semantically.

The CC text is just a sequence of words and does not have the prominent indicator of the scene change. Therefore, we consider the change of the speaker or the interval of the talk as the boundary of the CC text and define the segment between the boundaries as a CC segment. Each segment is supposed to belong to one of the scene categories.

Based on the structure of sports TV program, the scene categories we try to classify are the "Live" ("Live"+"PreLive" in Figure1), "Others-Game-related"("Post-Live"), "CM", and "Others-Game-unrelated" ("Report", "Studio", etc.) scenes.

Considering the structure of sports TV program, we notice that the scenes have some rules in how they line up. Therefore, the scene category of a CC segment depends on the scene category of the previous CC segment as well as its own features. In this paper, owing to the uncertainty of the information, we use a probabilistic framework which can handle such information, namely Bayesian Network(BN) [9], to categorize each CC segment.

The BN in Figure4 shows the relationship between the present scene and other factors. Node $B$ represents the category of the previous scene and is the parent of the node $X$, which represents the category of the present scene. Nodes $F_{j}$, which are the $j$ th children nodes of $X$, represent the features of the previous segment.

Based on this BN, we can calculate the probability of each value $x$ for the previous scene categories as

$$
P(x \mid e)=\left[\sum_{\text {alle }_{B}} P\left(x \mid e_{B}\right) P\left(e_{B}\right)\right] \prod_{j=1}^{|F|} P\left(e_{F_{j}} \mid x\right),
$$

where $e$ represents the values of variables on nodes other than $X, e_{F_{j}}$ and $e_{B}$ the values of the probabilities of each state of the corresponding nodes.

Bearing those discussed above in mind, we categorize each CC segment into the scene categories as shown below.

\section{[Procedure to categorize $\mathrm{CC}$ segment ]}

1. Calculate the conditional probability distributions(CP table) for every arc from the sample $\mathrm{CC}$ text.

2. Input the features of a $\mathrm{CC}$ segment and the scene category of the previous $\mathrm{CC}$ segment, then calculate the probability of each scene category for the segment based on the generated CP table and determine its scene category as the one which has the maximum value.

3. Normalize the calculated probability of each scene category, update the $P(b)$ with these values and repeat 2 . and 3 . for the rest of the segments.

After categorizing all CC segments, the story unit can be detected by identifying sequences between a live segment and the next live segment. Note that the live scenes can sometimes occur successively without any other in-between scenes. When there are consecutive live segments, we consider them as the consecutive separate live scenes if they have an interval more than a threshold between themselves. Otherwise, we determine them as included in the same live scene.

\subsection{Association with Video Stream}

In order to associate the text segments with the corresponding video segments, we have to find the story units also from the video stream. As discussed in the beginning of Section 3 , since the live scene usually starts with some specific images, taking some of these images as the example images and matching them with the beginning frames of each shot should find the beginning of the live scene, which is also the beginning of the story unit.

The "Timed-Stamp" in the CC text represents the rough time when they were spoken. Therefore, these stamps enable us to estimate the time when the sentences were spoken on the video. Moreover, since a human $\mathrm{CC}$ translator types what is said just after he/she hears the utterance in sports videos, the $\mathrm{CC}$ text lags behind the image stream ${ }^{1}$.

The video stream is a sequence of the camera shots each of which represents a continuous action in time and space. The boundaries of the shots which can be extracted from the image stream are considered to be more suitable for story based segmentation than the boundaries extracted from the text stream. Considering the features mentioned above, we associate the CC story units with the video story units as follows:

1. Search the CC story unit which starts around Th seconds after the beginning of the video unit.

2. If there are more than one $\mathrm{CC}$ unit before the beginning of the next video unit, associate with the closest one. Otherwise, divide the previously associated CC segment at the closest to Th seconds after the beginning of the video unit and change the scene category of the first scene unit of the divided segment to "Live".

\footnotetext{
${ }^{1}$ This is the characteristic of sports videos. The time difference between $\mathrm{CC}$ texts and video streams depends on the kind of videos.
} 


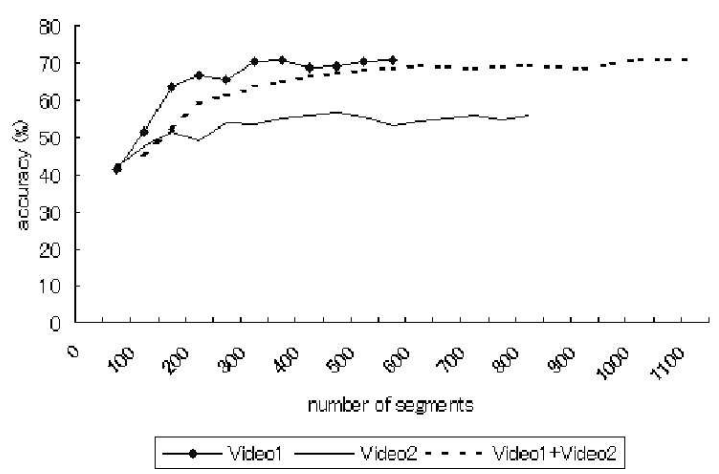

Figure 5: Results of Scene Segmentation

3. Change the scene category of the first scene unit of each remaining text unit to "Others-Game-related" and merge it with its previous story unit.

\section{EXPERIMENTAL RESULTS}

We tested our method for a broadcasted American football video(Video1), learning with the training data from the following three streams: 1)the same video stream(Video1), 2)another video stream(Video2), and 3)the joined one(Video1+Video2). Video1 and Video2 were about 2.5 and 3.5 hours in length, and contained about 550 and $850 \mathrm{CC}$ segments respectively. For the joined video stream, we combined the same number of segments extracted from each video.

Figure 5 shows the results of the scene segmentation of the $\mathrm{CC}$ text. We used 6 features as the features of a CC segment to test the idea: the name of the announcers, the number of the sentences (which represents the length of the segment), the players' names, the length of the sentences, the phrases representing the game situation (First and 10, etc.), and the numbers (which should represent the score, yards, etc.). The results are evaluated in terms of the accuracy defined as the ratio of the number of correctly categorized segments to total number of test data segments. These results tell us that

- About 400 training segments are enough to categorize the unknown text data.

- Although the BN learned from another video stream degrade the performance, learning partially from several videos should enable us to segment various videos of the same kind of sports.

We also detected the story units from the $\mathrm{CC}$ segmentation results. Table1 shows the results evaluated by the recall rate ( $\sharp$ of correct segmentation/ $\sharp$ of actual segmentation) and the precision rate( $\sharp$ of correct segmentation/ $\sharp$ of performed segmentation).

We experimented the association method only with the parts(about 1 hour) of Video1. We manually segmented the video stream into the story unit beforehand. After the association, we were able to exclude all the incorrectly extracted text story units, and also found the unit which we had failed to extract by only the text analysis.
Table 1: Results of Story Segmentation

\begin{tabular}{c|c|c|c}
\hline & Video1 & Video2 & Video1+2 \\
\hline Recall & $94 \%(140 / 149)$ & $82 \%(122 / 149)$ & $93 \%(138 / 149)$ \\
\hline Precision & $70 \%(140 / 201)$ & $70 \%(122 / 174)$ & $72 \%(138 / 192)$ \\
\hline
\end{tabular}

Our text segmentation method uses few domain-specific features and considered to be easily applicable to other kinds of sports. Also, since using several superficial features reduces the importance of the correctness of the CC text, the errors in the CC text did not have much effect on the performance. Although we segmented the video stream manually, the "Live" scene should be extracted with the image analysis as stated in $[7,8]$. We will deepen the consideration about the image analysis in our future work.

\section{CONCLUSION}

This paper proposed a model to represent a broadcasted sports video in a structural way on the basis of structures of a sports TV program and a sports game, and also a method to segment the video and the speech transcript into the semantical units for the representation. Though our segmentation method only tried to structurize the video as a repetition of story units, the results can be put to more practical use. For example, keyword extraction from those important scene units will help us to get the information about each story unit such as player's name, game situation, and so on. Furthermore, since our method makes a distinction among the CC segments on the basis of the importance for the story, picking up the segments according to their importance will enable us to make the sports speech summarization.

\section{REFERENCES}

[1] M.Lazarescu, S.Venkatesh, G.West, and T.Caelli, "On the Automated Interpretation and Indexing of American Football," Proc. IEEE ICMCS'99, vol.1, pp.802-806, 1999.

[2] Y.Chang, W.Zeng, I.Kamel, and R.Alonso, 'Integrated Image and Speech Analysis for Content-Based Video Indexing," Proc. IEEE ICMCS'96, pp.306-313, 1996.

[3] N.Babaguchi, Y.Kawai, and T.Kitahashi, 'Event Based Indexing of Broadcasted Sports Video by Intermodal Collaboration", IEEE Transaction on Multimedia, vol.4, no.1,pp.68-75, March 2001.

[4] Y.Nakamura and T.Kanade: 'Semantic Analysis for Video Contents Extraction - Spotting by Association in News Video.", Proc. of The Fifth ACM International Multimedia Conference, Nov 1997.

[5] N.Nitta, N.Babaguchi, and T.Kitahashi: 'Extracting Actors, Actions and Events from Sports Video - A Fundamental Approach to Story Tracking -",Proc. ICPR'O0, pp.718-721,2000.

[6] W.Zhu, and C.Toklu, and S.P.Liou, "Automatic News Video Segmentation and Categorization Based on Closed-Captioned Text",Proc. IEEE ICME'01,2001.

[7] D.Zhong, and S.F.Chang, "Structure Analysis of Sports Video Using Domain Models", Proc. IEEE ICME'01, 2001.

[8] P.Xu, L.Xie, S.-F.Chang, A.Divakaran, A.Vetro, and H.Sun, "Algorithms and System for Segmentation and Structure Analysis in Soccer Video", Proc. IEEE ICME'01,2001.

[9] R.O.Duda, P.E.Hart, and D.G.Stork,'Pattern Classifi cation", A WileyInterscience Publication. 